\title{
Generation of highly symmetric, cylindrically convergent shockwaves in water
}

\author{
S. N. Bland ${ }^{1}$, Ya. E. Krasik ${ }^{2}$, D. Yanuka ${ }^{2}$, R. Gardner ${ }^{1}$, J. MacDonald ${ }^{1}$, A. Virozub ${ }^{2}$, S. Efimov², \\ S. Gleizer ${ }^{2}$, and N. Chaturvedi ${ }^{1}$ \\ ${ }^{1}$ Institute of Shock Physics, Imperial College London, London SW7 2BW, UK \\ ${ }^{2}$ Physics Department, Technion - Israel Institute of Technology, Haifa 32000, Israel
}

\begin{abstract}
We report on pulsed power driven, exploding copper wire array experiments to generate cylindrical convergent shockwaves in water employing $\mu$ s risetime currents $>550 \mathrm{kA}$ in amplitude and with stored energies of $>15 \mathrm{~kJ}$ - a substantial increase over previous results. The experiments were carried out on the recently constructed $\mathrm{MACH}$ facility at Imperial College London in collaboration with colleagues from Technion, Israel. 10mm diameter arrays consisting of $60 \times 130 \mu \mathrm{m}$ wires were utilized and current and voltage diagnostics of the load region suggested $\sim 8 \mathrm{~kJ}$ of energy was deposited into the wires (and the load region close to the wires) during the experiments, resulting in them forming dense, highly resistive plasmas that rapidly expanded driving the shockwaves in the water. Laser-backlit framing images of the shock front were obtained at radii $<0.25 \mathrm{~mm}$ for the first time, and there was strong evidence that even at radii $<0.1 \mathrm{~mm}$ this front remains stable, resulting in a convergence ratio of $>50: 1$. Framing images and streak photography showed the velocity of the shockwave reached $\sim 7.5 \mathrm{kms}^{-1}$ at $0.1 \mathrm{~mm}$ from the axis. $2 \mathrm{D}$ hydrodynamic simulations that match the experimentally obtained implosion trajectory suggest pressures of $>1 \mathrm{Mbar}$ are produced within $10 \mu \mathrm{m}$ of the axis along with water densities $3 \mathrm{gcm}-3$ and temperatures of many 1000 of Kelvin. In these conditions QEOS suggests a strongly coupled plasma with an ionization fraction $\sim 0.7$ would be formed. The
\end{abstract}


results represent a 'stepping stone' in the application of the technique to drive different material samples into high pressure, warm dense matter regimes with compact, university scale generators, and provides support in the scaling the technique to multi-mega ampere currents.

\section{Introduction}

Production of highly symmetric, cylindrically convergent pressure waves is appealing for equation of state (EOS) research as they allow high pressures and densities to be reached in relatively compact systems. Several methods for producing convergent shockwaves have been developed, for instance a high explosive 'jacket ${ }^{1,2,3}$ or redirection of the planar compression produced in a gas-gun impact ${ }^{4}$. High current pulsed power has also been used to produce 'quasiisentropic' pressure waves, with the current flowing through the outer layers of a liner and the interacting with the induced magnetic field to produce compression of the liner ${ }^{5}$. Recent experiments on the world's largest pulsed power accelerator - the 26MA Z facility at Sandia National Laboratories - have used a highly shaped current pulse to produce quasi-isentropic compression of a liner with a cryogenic deuterium fill to the Gbar level, which was diagnosed with monochromatic X-ray radiography ${ }^{6}$.

The use of convergent geometries on smaller scale pulsed power generators has, so-far, been limited. Despite the potential advantages, issues with diagnostics and diagnostic access have restricted most EOS research to be planar in nature, typically utilizing magnetically driven strip lines to peak pressures of a few $10 \mathrm{~s}-100 \mathrm{~s}$ of $\mathrm{kbar}^{7,8,9}$.

Research at Technion has demonstrated a promising new method of producing convergent shockwaves in water that does not rely on magnetic compression, and instead utilizes the 
electrically driven explosion of arrays of metallic wires ${ }^{10,11}$. The shockwaves produced can be diagnosed optically over the majority of the radius of convergence (typically $5-10 \mathrm{~mm}$ ) and over durations of 100s of nanoseconds. Further, the technique places far less stress on the pulsed power generator than magnetic techniques as the load is critically damped i.e. a large fraction of the energy stored in the generator is used to explode the wires and produce the shockwaves, so there is no 'ringing'/large scale reversal of the current as often observed with inductive loads.

To produce the shockwaves, symmetric arrays of $\sim 100 \mu \mathrm{m}$ metallic wires are placed underwater and a sub- $\mu$ s rise time, several $100 \mathrm{kA}$ current is applied to the wires. The water acts to prevent breakdown of the wire material along its surface ${ }^{12,13}$ (as found in typical wire array z-pinch experiments in vacuum) and tamps the wires expansion, resulting in very large energies being deposited into the wire material through Ohmic heating - so far energies up to $200 \mathrm{eV}$ per atom has been measured with copper wires ${ }^{10}$. The entire volume of the wire undergoes phase changes, entering a dense, highly resistive gas-plasma $\mathrm{mix}^{14}$. The large energies deposited into the wire and its associated change in phase cause it to rapidly expand, launching a uniform, radially expanding shockwave into the surrounding water. With the wires arranged into a cylindrical array, shockwaves from adjacent wires rapidly merge together, forming a highly symmetric, many $\mathrm{kms}^{-1}$ implosion that converges onto the axis of the experiment.

Experiments at Technion have primarily explored cylindrical arrays driven by $\sim \mu$ s risetime currents with peaks up to $300 \mathrm{kA}$, which resulted in energies up to $4.5 \mathrm{~kJ}$ being deposited in the exploding wires; whilst in some experiments with much faster risetimes of 350ns and currents $500 \mathrm{kA}, \sim 6 \mathrm{~kJ}$ was deposited in the wires ${ }^{10,11}$. However, there has been little imaging of the 
shockwaves produced close to the axis of the array, with framing images at $\sim 0.2-0.3 \mathrm{~mm}$ radius only in the lower current experiments. Trajectories of the imploding shockwaves were measured from the framing data at high radii then inferred from a 'time of flight measurement' with the arrival of the shockwave at the axis set to correspond to the onset of increased optical emission seen on photodiodes. These trajectories were compared with hydrodynamics simulations incorporating a high pressure EOS for water and copper which indicated that at implosion of such a shockwave, $\sim$ Mbar pressures would be produced at radii $\sim 5 \mu \mathrm{m}$ from the axis, and that the temperature of the water would reach many 1000s of Kelvin - consistent with the optical emission seen to originate from the axis. The technique has now been extended to produce spherically symmetric implosions ${ }^{11}$ where there is good evidence that pressures $>10 \mathrm{Mbar}$ are produced close to the centre of the implosion. However, key questions related to the stability, symmetry and trajectory of the converging shockwaves at radii smaller than $\sim 0.2 \mathrm{~mm}$, and the possibility of operating such wire arrays with significantly larger deposited energies remain unanswered. Thus there is great interest in scaling experiments to larger currents/large driver energies, with better diagnostic techniques that can image the shockwaves closer to the axis.

This paper details the first underwater wire array research with $\sim \mu$ s risetime currents of amplitude $>500 \mathrm{kA}$. The experiments were carried out on the MACH generator at Imperial College London ${ }^{15,16}$ in collaboration with colleagues from Technion. In Section II of this paper the experimental set up and diagnostics used to measure the shockwaves are described. Section III includes results and discussion of the experiments. In the first part of the Section, IIIA, experiments with thick solid copper liners are detailed, which were primarily used to calibrate current and voltage diagnostics positioned relatively close to the load. Despite no large scale 
movement of the liner, a shockwave was observed to launch from its inner surface and converge on axis - the mechanism which formed this shockwave is still under study. In the second part of the Section, IIIB, experiments with cylindrical arrays of wires are described. Measurements of the energy resistively deposited into the wires are presented and used to provide evidence of the state of the wire material as the wires explode into dense, resistive, strongly coupled plasmas. Framing images show the imploding shock front driven by the wire expansion is highly uniform and there is strong evidence that convergence ratios $>50: 1$ are obtained. Simple calculations of the pressures at different radii are performed. In Section IV the results of hydrodynamic calculations of the pressure, density and temperature that the water attains in the final stages of the implosion are presented, based on the implosion trajectories measured in Section IIIB. Comparing these parameters with QEOS models suggests the formation of a high pressure, strongly coupled plasma on axis. Finally, Section V provides a summary, places the work in context with previous results, and briefly discusses future research areas.

\section{Experimental setup and diagnostics}

Experiments were performed on the Mega-Ampere-Compression-and-Hydrodynamics (MACH) pulsed power facility at Imperial College London. MACH is a $100 \mathrm{kV}$ cavity that was designed to produce $2 \mathrm{MA}$ currents with $\sim 450 \mathrm{~ns}$ risetimes when connected to a low inductance $(<5 \mathrm{nH})$ transmission line and load. The cavity was to be the basis of a much larger Linear Transformer Driver accelerator, however, as no other cavities were made, $\mathrm{MACH}$ has been configured as a set of 40 parallel capacitors in series with 20 low inductance switches that connect via. a radial feed. In its simplest form this can be represented as a 'lumped' $6 \mu \mathrm{F}$ capacitance in series with an inductance of $\sim 20 \mathrm{nH}$ and a resistance of $15 \mathrm{~m} \Omega$. 
For the experiments described in this paper, the capacitors were charged between 60 and $70 \mathrm{kV}-$ a stored energy of $10.8-14.7 \mathrm{~kJ}$ (the latter being almost twice the stored energy ever used in experiments at Technion). The inductance of the load and transmission line connected to the cavity were comparatively high $(\sim 25 \mathrm{nH})$, which strongly affected the systems overall performance - for instance in experiments with the solid metal liners described in section IIIA peak currents were reduced to $5-600 \mathrm{kA}$ and risetimes increased to $\sim 1000 \mathrm{~ns}$.

The load configuration used for the water shock experiments is shown in Fig. 1. The anode and cathode plates were separated by $\sim 8 \mathrm{~mm}$ during the experiments, and the entire region from the upper surface of cathode plate to the top of the current return cylinder was flooded with deionized water - immersing the wire array. Shock absorbing materials and spring loaded bolts were used throughout the load region, as the shockwaves transmitted through the water were highly damaging - regularly shearing fixings, and bending $10 \mathrm{~mm}$ thick stainless steel components such as the return current can.

The wire arrays used typically consisted of $60 \times 130 \mu \mathrm{m}$ copper wires, on a diameter of $10 \mathrm{~mm}$ and with a length of $40 \mathrm{~mm}$. These parameters were chosen based on a combination of previous experiments and hydrodynamics simulations performed at Technion, and preliminary experiments at Imperial College. The number of wires was chosen to provide good azimuthal symmetry - too few wires and the shockwaves from adjacent wires would not merge into the desired cylindrically symmetric, convergent shockwave travelling towards the axis. The diameter of the individual wires was selected to optimize the transfer of the energy supplied by MACH 
into heating of the wires and driving their subsequent explosion into a highly resistive gas-dense plasma mix. In practice this was achieved by altering the wire diameter so that the rapid increase in resistance associated with the explosion occurred at $70-80 \%$ of the time of peak current in a short circuit experiment. The length of the wires was chosen to ensure no low resistance plasma channels formed early in the experiment, for example through electrical breakdown along the surface of the wires, which would limit the heating and explosion of the wires and produce an underdamped oscillation in the current. Finally the diameter of the array was set at $10 \mathrm{~mm}$, to ensure that the convergent shockwave was well formed and approaching, but not yet reached, the axis by the time the all the possible energy had been transferred to wires.

Current and voltage through the load were measured via. two 'B-dot' magnetic pick-up coils and one 'D-dot' capacitive probe, all placed $15 \mathrm{~cm}$ from the axis of the array. Initial experiments utilizing thick, non-imploding copper cylinders (Section IIIA) enabled in-situ calibration of these measurements through comparison with a set of 'machine diagnostics' - large Rogowski coils placed close to the capacitors.

Dynamics of the shockwaves produced in the experiments were monitored via. laser shadowgraphy images along the axis of the array, and streak photography across a chord of this (Fig. 2). A $6.5 \mathrm{~W} 532 \mathrm{~nm}$ laser provided backlighting to the shadowgraphy system, and was fiber coupled to beneath the load region - preventing any damage to the laser from shockwaves transmitted through $\mathrm{MACH}$. Light from the fiber was collimated into a $\sim 20 \mathrm{~mm}$ diameter beam before being fed through the array; then after the array was image relayed to a small optical table. Here the light was split into an Invisible Vision framing camera with twelve 1024x1024 
pixel frames of $5 \mathrm{~ns}$ exposure and a Kentech $40 \mathrm{~mm}$ optical streak camera backed with a digital single lens reflex camera. Different magnifications, frame timings and streak durations were used in the experiments to highlight both the gross dynamics of the shockwaves and the convergence closer to axis. Calibration of the exposure times and streak times were performed via. a high speed LED pulser; whilst magnifications were calibrated using test objects (e.g. the 1951 USAF resolution slide) placed at the midpoint of the array.

\section{Results and Discussion}

\section{IIIA Calibration experiments with thick copper liners}

In order to calibrate the B-dot and D-dot diagnostics close to the load, several experiments were performed using thick copper liners to act as loads of low resistance/constant inductance. Ignoring any capacitive effects in the load region, the current monitored via. the B-dots could then be directly compared to measurements by the machine diagnostics. Meanwhile the voltage across the load measured by the D-dot could be compared with:

$$
V=I R+L \frac{d I}{d t}+I \frac{d L}{d t}
$$

The liners used in these experiments had an outer diameter of $9.5 \mathrm{~mm}$ and a length $\sim 50 \mathrm{~mm}-$ similar to the diameter and length of the arrays used in subsequent experiments. The wall thickness of the liner was $\sim 0.9 \mathrm{~mm}$ and after an experiment there was no obvious change in the outer/inner radii. Neither was there any sign of damage to the inner or outer surfaces, suggesting any heating of the liner was to well below its melting point.

A typical current pulse through the liner measured by the machine diagnostics is shown in Fig. 3a. The pulse has a peak 540kA @1010ns after the start of the current pulse and is lightly 
damped. We can use this to estimate the importance of the terms on the right hand side of Eq. 1. Knowing the liner does not move, we can assume the last term tends to zero. Given the liner remains solid and knowing the frequency of the current pulse $(\sim 0.25 \mathrm{MHz})$, the resistive first term will be much less than the inductive second term throughout the majority of the pulse except when $\mathrm{dI} / \mathrm{dt}$ tends to zero at current maxima or minima. Hence we can use $\mathrm{V}=\mathrm{L} \mathrm{d} / \mathrm{dt}$ during the rising edge of the current pulse to calibrate the voltage measured by the D-dot diagnostic as shown in fig. $3 \mathrm{~b}$. Here the inductance was calculated to be $\sim 27 \mathrm{nH}$ from the geometry of load region, which compared well with estimates of the inductance made by circuit analysis $(25-29 \mathrm{nH})$ that matched the period of the current waveform in the experiment.

Despite there being no obvious change in the size of the liner after the experiment, laser shadowgraphy images along the axis show a shockwave is still launched from the inside surface of the liner (Fig. 4). The shock is launched at $\sim 460 \mathrm{~ns}$ into the current pulse, then proceeds towards the axis at a constant velocity of $\sim 1.8 \mathrm{kms}^{-1}$. The shadowgraphy images demonstrate the shock front remains symmetric, with the final image at a radius of $0.8 \mathrm{~mm}$ and the implosion carrying on beyond this.

The precise mechanism through which the shock is launched from the liner is still being examined. The skin layer in which the current flows $(=\sqrt{2 / \sigma \omega \mu}$ where $\sigma$ is conductivity, $\omega$ angular frequency and $\mu$ permeability) will initially be $\sim 0.1 \mathrm{~mm}$, much less than the liner thickness of $\sim 0.9 \mathrm{~mm}$. As heating of the liner is limited, this is unlikely to increase significantly on the time scale of the experiment, and current will not reach the inner surface. Similarly the time taken for conduction to heat the inner surface to $>100^{\circ} \mathrm{C}$ and cause boiling of the water will 
also likely be longer than the timescale over which the shockwaves are observed - besides which boiling is not usually a uniform process, so might not explain the symmetry of the shock front. A more feasible explanation is the transmission of an increasing pressure wave through the liner into the water. The interaction of the current with its induced magnetic field will result in magnetic pressure, compressing the liner. Given the elastic sound speed in copper, $\sim 4760 \mathrm{~ms}^{-1}$, this pressure wave will take $\sim 190$ ns to transmit through the liner wall; hence the time at which the shockwave is launched from the inner wall corresponds to the pressure at a time of $460 \mathrm{~ns}-$ $190 \mathrm{~ns}=\sim 270 \mathrm{~ns}$ at the outside. At this point the current is $\sim 190 \mathrm{kA}$ and so the magnetic pressure $\left(=\mathrm{B}^{2} / 2 \mu\right)$ is expected to be $\sim 0.3 \mathrm{kbar}$. Whilst not high, the impedance mismatch between the copper and water could result in a rapid ramp up of the pressure wave into the observed shockwave. We note also that at peak current the magnetic pressure will be only $\sim 2.6 \mathrm{kbar}$, which is comparable to the yield strength of copper (typically measured $\sim 0.7-3 \mathrm{kbar}$ depending on how the copper has been formed/alloyed), and so the liner could be behaving elastically, not plastically, resulting in the lack of any measurable change to the liner inner/outer diameter after an experiment.

Whilst not being as fast as the shockwave that reaches the axis when launched from wire arrays (as described in the following Section) the phenomena of shockwaves launched from the inner surface of the liner could also have specific uses. For instance, as the liner remains intact during the experiment, the system could be coupled to a repetitive pulsed power generator and used to launch multiple smaller shockwaves through media for materials processing or even food $\operatorname{preparation}^{17}$. 


\section{IIIB Experiments with wire arrays}

The current through and voltage across a typical experiment utilizing a wire array load is shown in Fig. 5a. There are immediate differences compared with the liner experiments. In particular the current shows a peak of $\sim 500 \mathrm{kA}$ at only $620 \mathrm{~ns}$, and demonstrates little sign of reversal - i.e. the resistance of the wires as they heat up and undergo phase changes has critically damped the current pulse.

Returning to Eq. 1 , and assuming that the $3^{\text {rd }}$ term remains zero (i.e. for the duration of the experiment current remains concentrated at/close to the original position of the wires) we have $\mathrm{V}$ $=\mathrm{IR}+\mathrm{L} \mathrm{dI} / \mathrm{dt}$. Using a calculated value for the inductance of the array $(\sim 25 \mathrm{nH}$, similar to that used in the liner experiments) we can use the current measured in the experiment to split the voltage signal into resistive and inductive components, as shown in Fig. 5b. This demonstrates that the resistive part of the voltage signal spikes to $60-65 \mathrm{kV}$ just after peak current i.e. when the inductive component is minimal.

These measurements enable the state of the material from the wires to be determined. Integrating the resistive component with the current through the array $\int \mathrm{IV}_{\text {resistive }} \mathrm{dt}$ gives the energy dissipated in the array as $8 \pm 0.7 \mathrm{~kJ}$ (Fig. $5 \mathrm{c}$ ), where the error is determined by a combination of noise, uncertainties in the inductance, and hence uncertainties in the calibration of the D-dot probe. At peak voltage, the resistance of the array is $\sim 0.12 \Omega$, so the resistance of each wire has reached $\sim 7 \Omega$. This is significantly more than the initial value of $0.05 \Omega$ expected at room temperature. The current and resistive voltage were used as inputs to high resolution MHD simulations of a wire/surrounding water to calculate the temperature and density of the wire 
material - such simulations have been calibrated against previous single wire data from Technion ${ }^{14}$. The temperature of the wire is shown along with the dissipated energy in Fig 5c, and suggests that melting would start at $\sim 280 \mathrm{~ns}$, and boiling at $\sim 400 \mathrm{~ns}$. Throughout this time resistance is increasing, initially in a linear fashion with temperature whilst the wire is solid, and then exponentially as the wire turns into a liquid. At the peak of energy deposition, calculations suggest a temperature of $22000 \mathrm{~K} / 2 \mathrm{eV}$ is reached and the copper will have an ion density $\sim 5 \times 10^{20}$ $\mathrm{cm}^{-3}$. A Quotidian Equation of State (QEOS) model of the copper, based on the Franfurt model ${ }^{18}$, suggests that at this point the ionization fraction will be $\sim 0.5$ i.e. a partially ionized, strongly coupled plasma has been formed.

Shadowgraph images along the axis of the array show a highly symmetric, cylindrically converging 'shockwave' travelling towards the axis - see Fig. 6. Tracking the position of the shock front with time demonstrates that this appears to have been launched relatively early in the experiment within $\sim 50 \mathrm{~ns}$ of the start of the current pulse. Until it reaches a radius of $2.5 \mathrm{~mm}$, at a time of $1.7 \mu$ s (i.e. long after the peak current), the velocity of the shock front remains at $\sim 1.5 \mathrm{kms}^{-1}$. This corresponds to the speed of sound in water, suggesting little energy will be required to drive this weak 'shockwave' or sonic wave. A similar weak shockwave/sonic wave launched early in time has been obtained in both single wire and wire array studies at Technion ${ }^{10}$. There are several possible explanations to how this wave is formed. It could be due to the still solid copper wires undergoing elastic-plastic transitions driven by magnetic pressure even at only 50ns into the current pulse, the field around the wires will already have a magnetic pressure $\sim 10 \mathrm{kBar}$, well above the yield strength of copper. Alternately the increased magnetic field around the wires (compared with that around the liners) might result in magnetic diffusion 
becoming highly non-linear and current melting the surface of the wires earlier than expected. Finally, the surface of any wire will always have some contamination/oxidation and this could rapidly vaporise.

At $\sim 1.7 \mu$ s the velocity of the shock front suddenly increases to $\sim 3.5 \mathrm{kms}^{-1}$, and this new velocity remains constant until a radius $\sim 0.5 \mathrm{~mm}$. Higher magnification images, such as those shown in fig. 7 demonstrate that throughout this time the shock front remains highly uniform and symmetric. Tracking the trajectory of the shock front back to the radius of the wire array suggests that this higher speed is due to a shockwave launched when the wires are expected to boil, ionize and explode and energy deposition into the array rapidly increases.

As the shock front gets within $\sim 0.3 \mathrm{~mm}$ of the axis measurements of symmetry become more challenging as laser speckle - the mottle pattern seen in Figs. 6 and 7 - becomes difficult to account for, and any small deviations in the light from the probing laser could easily result in images being clipped. Nevertheless images (fig $7 \mathrm{~d} / \mathrm{e}$ ) show a near circular pattern of light being transmitted along the axis of the array down to a radius of $0.1 \mathrm{~mm}$ - a $50: 1$ radial convergence.

In several experiments laser-backlit optical streak photography across a chord of the array was used to supplement the shadowgraph images over the last $\sim 2 \mathrm{~mm}$ radius of the array. The position of the shock front recorded in the streaks - e.g. fig. $8 \mathrm{a}$ - overlaid measurements made via. the shadowgraph images. Within a radius of $0.5 \mathrm{~mm}$, both streak photography and shadowgraphs show that the shock front continually accelerates, reaching a velocity $\sim 7.5 \mathrm{kms}^{-1}$ at $0.1 \mathrm{~mm}$ 
radius. This acceleration is due to the pressure behind the shock front increasing with the decrease in cross sectional area of the shock ${ }^{19,20}$.

When/shortly after the shock reaches the axis, there is a small increase in the intensity recorded across the entire radius of the streak image. This most likely corresponds to the rapid heating and ionization of material on axis, which will emit into $4 \pi$ along the length of the array (so is not localized on the streak image). Such an increase in emission has been seen in photodiodes collimated to view the axis of the array in experiments at Technion ${ }^{10}$, where the increase in emission is used to determine the time of flight of the shockwave.

We can use observations of the implosion trajectory to estimate the pressure behind the shockwave. As discussed in references 19 and 21, a self-similar solution to the implosion of a shockwave can be found by relating the radius of shock front to the time using $R\left(t_{i m p}-t\right)=A\left(t_{i m p}\right.$ - $t)^{\alpha}$ where $t_{\text {imp }}$ is the time of implosion, $A$ is a constant determined by initial conditions and the method of shockwave generation, and $\alpha$ is a factor that depends on geometry which equals 0.75 for cylindrical systems. Using the conservation laws of momentum and energy, and assuming the shockwave is strong, enables the pressure immediately behind the shock front to be estimated $\operatorname{as}^{19,21}$ :

$$
P=2 \frac{\rho_{\mathrm{o}} \mathrm{U}_{\mathrm{S}}^{2}}{(\gamma+1)}
$$

where $U_{s}$ is the shock velocity, $\rho_{0}$ the initial water density and $\gamma$ is the adiabatic index. At $0.1 \mathrm{~mm}$ radius, using $\mathrm{U}_{\mathrm{s}}=7.5 \mathrm{kms}^{-1}$ and $\gamma=7.15$, this suggests the pressure is $\sim 140 \mathrm{kbar}$. The similarity approach then estimates the pressure behind the shock front at a radius of $10 \mu \mathrm{m}$ as being:

$$
\mathrm{P}_{10 \mu \mathrm{m}}=\mathrm{P}_{100 \mu \mathrm{m}}\left(\frac{10 \times 10^{-6}}{100 \times 10^{-6}}\right)^{2-\frac{2}{\alpha}}
$$


Hence at $10 \mu \mathrm{m}$, providing the shock remains stable and uniform we would expect a pressure of $\sim 650 \mathrm{kbar}$ behind the shock. Below a radius of $\sim 10 \mu \mathrm{m}$, simulations suggest radiation from heated material behind the shockfront becomes important, and it is difficult to continue using the same self-similar approach.

\section{Simulations of the shockwave implosions}

2D $r-\theta$ Lagrangian hydrodynamics simulations were compared with the trajectory of the shockwave to estimate the parameters including the pressure, density, temperature and energy density in the water behind the shock ${ }^{22,23}$. The simulations were based on the finite volume method and included conservation of mass, momentum and energy coupled with high pressure equations of state for copper and water.

The array parameters $(60 \times 130 \mu \mathrm{m}$ copper wires on a diameter of $10 \mathrm{~mm}$ and of length $40 \mathrm{~mm})$ were used as an input to the calculations, along with the measured power dissipated in the array found from $\mathrm{IV}_{\text {resistive }}$. For efficiency only $1 / 4$ of the array was simulated with periodic boundary conditions. A Delaunay triangulation method was used, with an initial mesh that varied from a characteristic scale of $\sim 60 \mu \mathrm{m}$ in the vicinity of the wires down to $\sim 5 \mu \mathrm{m}$ close to the axis. Each triangle in the mesh contained information of the pressure, temperature, density and specific energy, and the nodes contained information on the position, velocity and acceleration. Numerical convergence tests were performed to ensure the simulated values were stable.

In order to match the implosion trajectory of the shockwave within the last $\sim 2 \mathrm{~mm}$ of the radius only $\sim 75 \%$ of the input power calculated from $\mathrm{IV}_{\text {resistive }}$ was used in the simulations. A higher 
value and the implosion time would be significantly faster and vice-versa. This is consistent with previous results at Technion and the $25 \%$ difference represents several factors: $\sim 5-10 \%$ may be accounted for by resistive losses in the connections of the electrodes (which are also measured by the current and voltage probes); there are small systematic errors in the fit to the $\mathrm{IV}_{\text {resistive }}$ data in the simulations, again at the $5-10 \%$ level; and the remainder of the difference is due to small uncertainties in the implosion trajectory and losses through lower resistivity plasma at the interface of the exploding wires and the water.

The results of the simulations are shown in Fig. 9. The simulations suggest that at the time the shockwave reaches the axis, the water behind the shockwave will attain a pressure $>1 \mathrm{Mbar} / 100 \mathrm{GPa}$, and this will continue to rise closer to the axis. The density of the water will

reach nearly $3 \mathrm{~g} / \mathrm{cc}, 3 \mathrm{x}$ its standard value; whilst the temperature will reach many $1000 \mathrm{~s}$ of Kelvin, with $>10000 \mathrm{~K}$ on axis - these conditions are consistent with the sudden burst of optical emission observed by the streak camera shortly after the implosion occurs. The energy density, in the water, meanwhile, becomes $30-40 \mathrm{MJ} / \mathrm{kg}$. Given the high pressures produced, a QEOS model was again used to calculate the ionization states from the conditions predicted in the simulations. This suggested that at the time of the implosion, the hydrogen in the water would attain an ionization fraction of 0.7 within a radius at $10 \mu \mathrm{m}$, and later in time, as the stagnated material releases the ionization fraction at $50 \mu \mathrm{m}$ radius would reach similar values.

\section{Summary, comparisons to previous data \& future research areas}


We have successfully performed the first pulsed power driven, exploding underwater wire array experiments utilizing $\sim \mu$ s currents $>500 \mathrm{kA}$ and with driver energies of $>15 \mathrm{~kJ}$. The energies deposited into the wires during these experiments was determined to be $\sim 8 \mathrm{~kJ}$.

Shadowgraph images of the implosion demonstrate it remains stable and uniform until at least $0.35 \mathrm{~mm}$ radius, and we have obtained the first images of the implosion below $0.2 \mathrm{~mm}$, producing strong evidence that the implosion remains stable until $<0.1 \mathrm{~mm}$ from the axis, a compression ratio of at least 50:1.

The implosion trajectory of the shock front shows 2 distinct parts. For the majority of the experiment, until it reaches a radius of $\sim 2 \mathrm{~mm}$ the shock front has a velocity of only $1.5 \mathrm{kms}^{-1}$. It would appear this initial weak shockwave/sonic wave is launched at close to the start of the experiment. From a radius of $\sim 2 \mathrm{~mm}$ the shock front suddenly increases in velocity to $\sim 3.5 \mathrm{kms}^{-1}$. This is associated with a much stronger shockwave launched from the wires, due to them undergoing very rapid ohmic heating and converting to an expanding vapor-plasma mix. Within the last $0.5 \mathrm{~mm}$ radius the implosion trajectory is seen to continue to accelerate towards the axis, due to the effects of convergence. Using a combination of shadowgraphy images and streak photography, velocities of $\sim 7.5 \mathrm{kms}^{-1}$ were measured at $0.1 \mathrm{~mm}$ from the axis.

2D numerical simulations that match the implosion trajectory suggest pressures of $>1 \mathrm{Mbar}$ are produced within $10 \mu \mathrm{m}$ of the axis, with water densities $3 \mathrm{~g} / \mathrm{cc}$ and temperatures of many $1000 \mathrm{~s}$ of Kelvin. In these conditions QEOS calculations suggest that the water will become partially 
ionized, forming a strongly coupled plasma. Plasma in such conditions is often referred to as warm dense matter, and typical experiments to produce and analyse plasmas in these conditions usually take place at large National facilities.

Comparing the results of these experiments to previous work for scaling purposes can prove difficult due to the multiple different pulsed power generators previously utilized and the design of the arrays being varied to couple to these generators - with typically smaller wires being used at lower currents, and smaller diameter arrays at faster current risetimes. However, we can draw some simple conclusions:

i) Until these experiments, the greatest deposited energies with arrays driven by similar rise-time currents was $4.5 \mathrm{~kJ}$; and at shorter risetime (350ns) currents was $6 \mathrm{~kJ}$. Prior to this research one of the biggest concerns was whether significantly increasing the current/energy deposited into the wires would adversely affect the properties of the wires as they expanded into dense, highly resistive plasma columns - this was particularly worrying at longer current rise times as breakdown processes are stochastic. This does not appear to be the case, which bodes well for the use of the technique with larger, higher energy generators at multi-MA currents to produce yet higher peak pressures.

ii) Despite the high current and relatively long risetimes, the first images of the imploding shockwave at radii of $\sim 0.1 \mathrm{~mm}$ (half that previously achieved) provide strong evidence that the shock front remains stable. The trajectory of the implosion has also been directly measured at these radii for the first time, showing the trajectory behaves as expected from simulations, with a rapid acceleration in close proximity to the axis. 
iii) In some of the closest experiments to our conditions ${ }^{24}$ - with arrays of $40 \times 100 \mu \mathrm{m}$ copper wires on diameters of $10 \mathrm{~mm}$ and lengths of $40 \mathrm{~mm}$, driven by currents of $250 \mathrm{kA}-\mathrm{a}$ combination of experimental measurements at larger radii, time of flight data and simulation suggested the velocity of the shockwaves was $\sim 3.4 \mathrm{kms}^{-1}$ at $300 \mu \mathrm{m}$ radius and $5.5 \mathrm{kms}^{-1}$ at $50 \mu \mathrm{m}$ radius. Both are lower than the measured velocity in our experiments, which is $\sim 4.4 \mathrm{kms}^{-1}$ at $300 \mu \mathrm{m}$ radius, and even at $100 \mu \mathrm{m}$ is $\sim 7.5 \mathrm{kms}^{-1}$ and is continuing to accelerate. As the velocity is significantly higher, we would then expect a higher pressure/density/temperature plasma to be produced on axis.

iv) In the experiments described in (iii) above only $\sim 1 \mathrm{~kJ}$ was measured as being deposited into the wires, so with 'simple' scaling arguments (a factor of $\mathrm{x} 8$ in deposited energy) one would expect $\sim 3 \mathrm{x}$ the shock velocity seen in (iii) to be observed in our experiments. In reality, scaling is far more difficult due to the compressibility of the water as the shockwave travels through it. We note though that by using the same simulations used for experiments at lower currents on Technion, and with the same input parameters to these simulations (a fraction of deposited power), we are able to recreate the implosion trajectory of the shockwave and the time of arrival on axis. This again enables confidence in using the same simulations to plan for experiments at much higher currents and with different rise times.

Over the next few years the research will be extended in several ways. Whilst there is now good evidence to show that the use of underwater wire arrays will scale to higher currents/larger driver energies it is clear that further data is required in these conditions - in particular experiments must be performed to optimize array design here. We note here that despite MACH having a stored energy of $\sim 15 \mathrm{~kJ}$ only $\sim 8 \mathrm{~kJ}$ was deposited in the wires. This is less than the best 
experiments at Technion, in which drivers with a stored energy of $8 \mathrm{~kJ}$ deposited $6 \mathrm{~kJ}$ into the wires. To explore this, new load designs and transmission line hardware on MACH will significantly reduce its inductance, increasing the current in future experiments to the megaampere level whilst reducing the rise time of the current pulse. Simultaneously improvements in the diagnostics - both electrical and optical - are being planned to increase the accuracy of current and voltage data and enable the trajectory of the shockwave to be more readily followed to smaller radii. To diagnose the state of the material produced on axis, meanwhile, a combination of time and space resolved optical spectrometry and high-resolution phase contrast X-ray probing is being arranged, the later either being provided by a separately driven X-pinch ${ }^{25}$ and/or through transport of MACH to a synchrotron facility.

In addition to cylindrical experiments in water, we will explore the use of other materials, including plastics and cryogenic gases. Here the questions of whether a highly resistive plasma channel can still be obtained from the wires will be paramount.

Finally we will study different array geometries. The use of spherical arrays in water has been explored at Technion, resulting in far higher pressures expected on axis. Already the possibility of using these spherical implosions in water to drive small bubbles of deuterium/tritium fuel for neutron production has been discussed ${ }^{21}$. Exploring the use a spherical array directly in a cryogenic deuterium bath could prove to be less complicated route to neutron production and/or would enable estimates of the fusion cross section to be made in this high pressure, high density but low temperature parameter space where quantum tunneling and coulomb screening effects become increasingly important ${ }^{26}$ and present estimates of the cross section vary from $\sim 1 \times 10^{-35}$ to 
$1 \times 10^{-50} \mathrm{~cm}^{-2}$. Spherical arrays though - even more so than cylindrical systems - limit access for diagnostics. Hence we will explore new geometries such as hemi-spherically bent wires to enable the shockwaves to be more readily studied and to enable their use in driving separate experiments. Work will also commence on producing quasi-isentropic compressions by launching a succession of shockwaves from differently spaced wires.

Acknowledgements This work was supported by the Institute of Shock Physics, funded by AWE Aldermaston, and the NNSA under DOE Cooperative Agreement Nos. DE-F0302NA00057 and DE-SC-0001063. 


\section{Figure Captions}

Fig. 1(a) Diagram of the load region on $\mathrm{MACH}$ showing connections to the wire array in its holder, together with current (B-dot) and voltage (D-dot) probes. Purple arrows show the direction of conventional current flow - along the anode plate, then the inner surface of the current return cylinder, before travelling through the array holder and array, and heading back along the cathode plate. The anode plate, return cylinder and cathode are all made of stainless steel. Note that the D-dot probe is attached to the cathode plate in the same manner as the B-dot probes and at the same distance from the axis, but is 'into the page' in the diagram. (b) Picture of typical wire array in holder used in the experiments.

Fig. 2 Schematic of axial diagnostics used in the experiments - CW laser backlit (shadowgraphy) framing images and radial streak photography.

Fig. 3(a) Current through solid metal liner used in short circuit tests and trajectory of the shock front travelling from the inside surface of the liner towards the axis as measured from shadowgraph images. (b) Current measured from Bdot probes near the liner compared with that measured by machine diagnostics (Rog), and Voltage measured by Ddot probe near the liner compared with 'inductive' voltage calculated from current measurement assuming a constant inductance of $27 \mathrm{nH}$.

Fig. 4 Shadowgraph images showing the shockwave travelling from the inside surface of the liner towards the axis at (a) 100ns (b) 600ns (c) 1099ns (d) 1600ns and (e) 2100ns after the start of current. All axis are in mm. The shock front is highlighted by the red dotted circle. 
Fig. 5 (a) Current flowing through the array measured by machine diagnostics and voltage across array measured by Ddot probe. (b) Close up of first peak of current along with voltage measurement from Ddot, 'inductive voltage' calculated from current signal assuming a constant inductance and 'resistive voltage', the difference between the measured voltage and the inductive voltage calculation. Note the small rise and fall seen the resistive voltage around $100 \mathrm{~ns}$ is due to noise in the early time measurements of voltage from the Ddot probe (c) Calculation of energy deposited resistively into array during experiment $\left(=\int \mathrm{IV}_{\text {resistive }} \mathrm{dt}\right)$, along with temperature of wire material from high resolution simulations using measured current and voltage from experiment as inputs.

Fig. 6(a) - (f) Shadowgraph images of shockwave imploding towards the axis of the array @289ns, 499ns, 919ns, 1339ns, 1759ns and 2179ns after the start of current respectively. The shock front is highlighted by the red dotted circle and the axis are all in $\mathrm{mm}$. (g) Current through the array and trajectory of shock front travelling towards the axis as measured from the images.

Fig. 7 Higher magnification shadowgraph images of the shockwave at the centre of the array at (a) $1448 \mathrm{~ns}$ (b) $1748 \mathrm{~ns}$ (c) $1898 \mathrm{~ns}$ and (d) 2048ns after the start of the current pulse. The shock front is highlighted by the red dotted circle and the axis are all in mm. (e) Shows a zoomed in view of (d), with a $200 \mu \mathrm{m}$ diameter circle overlaid.

Fig. 8 (a) Laser-backlit streak photograph of the central part of an array showing the shockwave arriving on axis. The red dashed box shows an area of the image with enhanced brightness and 
contrast demonstrating an increase in intensity is observed at/just after arrival of the shock. (b) Trajectory of shock front as measured from shadowgraph images and streak photography. (c) Velocity of the shock front vs. radial position derived by fitting a trajectory to the images and streak data.

Fig. 9 Simulations of the experimental conditions made with a 2D Lagrangian hydrodynamics code (a) is the pressure in the water (b) is the density of the water (c) is its temperature and (d) the energy density in the water, all vs. time at different distances from the axis. 


\section{References}

${ }^{1}$ V. E. Fortov, R. I. Ilkaev, V. A. Arinin, V. V. Burtzev, V. A. Golubev, I. L. Iosilevskiy, V. V. Khrustalev, A. L. Mikhailov, M. A. Mochalov, V. Ya. Ternovoi, and M. V. Zhernokletov, Phys. Rev. Lett. 99185001 (2007).

${ }^{2}$ G.R. Fowles and W.M. Isbell, J. App. Phys 61377 (1965).

${ }^{3}$ Used by Z. Gu, H. Luo, H. Zhang, S. Zhao, X. Tang, Y. Tong, Z. Song, F. Tan, J. Zhao and C. Sun, Journal of Physics: Conference Series 500142018 (2014).

${ }^{4}$ A modification of the scheme described in D.R. Jones, D.E. Eakins, P. J. Hazell, D.J. Chapman and G.J. Appleby-Thomas in AIP Conf. Proc. 1426, 1141-1144 (2012).

${ }^{5}$ Such as those described in M. R. Martin, R. W. Lemke, R. D. McBride, J. P. Davis, D. H. Dolan, M. D. Knudson, K. R. Cochrane, D. B. Sinars, I. C. Smith, M. Savage, W. A. Stygar, K. Killebrew, D. G. Flicker, and M. C. Herrmann, Phys. Plasmas 19, 056310 (2012).

${ }^{6}$ P. F. Knapp, M. R. Martin, D. H. Dolan, K. Cochrane, D. Dalton, J.-P. Davis, C. A. Jennings, G. P. Loisel, D. H. Romero, I. C. Smith, E. P. Yu, M. R. Weis, T. R. Mattsson, R. D. McBride, K. Peterson, J. Schwarz, and D. B. Sinars, Phys. Plasmas 24, 042708 (2017).

${ }^{7}$ J. R. Asay, T. Ao, J.P. Davis, C. Hall, T. J. Vogler, and G. T. Gray, J. App. Phys. 103, 083514 (2008).

${ }^{8}$ G. Avrillaud, L. Courtois, J. Guerre, P.L. Hereil, F. Lassalle, F. Bayol, P. L'Eplattenier, B. Kovalchuck, E. Kumpjak, N. Zoi, and A. Kim, 14th IEEE International Pulsed Power Conference digest of technical papers Vol.2 P913-916 (IEEE Cat. No.03CH37472) (2003). 
${ }^{9}$ C. Sun, G. Wang, C. Liu, J. Zhao, F. Tan, G. Wang, J. Mo, N. Zhang, J. Jiang, and J. Chen, Proceedings of the Conference of the American Physical Society Topical Group on Shock Compression of Condensed Matter, AIP 978-0-7354-0469-4/07/1196 (2007).

${ }^{10}$ Ya. E. Krasik, A. Grinenko, A. Sayapin, S. Efimov, A. Fedotov, V. Tz. Gurovich, and V. I. Oreshkin, IEEE Trans. Plasma Sci. 36, 423 (2008) and references therein.

${ }^{11}$ Ya. E. Krasik, S. Efimov, D. Sheftman, A. Fedotov-Gefen, O. Antonov, D. Shafer, D. Yanuka, M. Nitishinskiy, M. Kozlov, L. Gilburd, G. Toker, S. Gleizer, E. Zvulun, V. Tz. Gurovich, D Varentsov, and M. Rodionova, IEEE Trans. Plasma Sci. 44, 412 (2016) and references therein.

${ }^{12}$ A. Grinenko, V. Tz. Gurovich, and Ya. E. Krasik, J. Appl. Phys. 100, 113309 (2006).

13 A. Fedotov, D. Sheftman, V. Tz. Gurovich, S. Efimov, G. Bazilitski, Ya. E. Krasik and V. I. Oreshkin Phys. Plasmas 15, 082704 (2008).

${ }^{14}$ D. Sheftman, D. Shafer, S. Efimov, and Ya. E. Krasik, Phys. Plasmas 19, 034501 (2012).

${ }^{15}$ S.N. Bland, K.H. Kwek, K. Omar, S. Stafford, J. Winters, and G. Wang, Bulletin APS Topical Conference on the Shock Compression of Matter 2015, Bull. Am Phys. 60 No 8 Abstract 10.1103/BAPS.2015.SHOCK.D1.3 (2015).

${ }^{16}$ S.N. Bland, K.H. Kwek, R. Gardner, D. Hinshelwood, G. Burdiak, K. Omar, J. Skidmore, R.B. Spielman, S. Stafford, J. Winters and G. Wang "MACH: a 2MA generator for compression experiments" in preparation for Phys Rev. Accelerators and Beams.

${ }^{17}$ F. Garcia, R. Woodall, US patent 6264543 B1, filed 3 Jul 2000.

${ }^{18}$ As developed by Dr Steven Faik of Frankfurt University, with code details/download packages available from http://th.physik.uni-frankfurt.de/ faik/feos.php?lang=eng (accessed 12/04/2017) 
${ }^{19}$ Y. B. Zeldovich and Y. P. Raizer, "Physics of Shock Waves and High-Temperature Hydrodynamic Phenomena, 2nd ed." (Academic, New York, 1967), Vol. 2.

${ }^{20}$ G. B. Whitham, "Linear and Nonlinear Waves" (John Wiley \& Sons, Inc., New York, 1974).

${ }^{21}$ A. Grinenko, V. Tz. Gurovich, and Ya. E. Krasik, Phys. Plasma 14, 012701 (2007).

${ }^{22}$ I.V. Oreshkin, R. B. Baksht, A. Yu. Labezkii, A. G. Rousskikh, A. V. Shishlov, P. R. Levashov, K. V. Khishchenko, and I. V. Glazyrin, Tech. Phys. 49, 843 (2004).

${ }^{23}$ A. Grinenko, Ya. E. Krasik, S. Efimov, A. Fedotov, V. Tz. Gurovich, and V. I. Oreshkin, Phys. Plasmas, 13, 042701 (2006).

${ }^{24}$ S. Efimov, A. Fedotov, S. Gleizer, V. Tz. Gurovich, G. Bazalitski, and Ya. E. Krasik, Phys Plasmas 15112073 (2008).

${ }^{25}$ S. A. Pikuz, D. B. Sinars, T. A. Shelkovenko, K. M. Chandler, D. A. Hammer, G. V. Ivanenkov, W. Stepniewski, and I. Yu. Skobelev, Phys. Rev. Lett. 89 035003-1 (2002).

${ }^{26}$ As discussed in report AD-A 214010 "Tunneling Effects on Low Energy Fusion Cross Sections" by C.L. Leakeas, prepared for Astronautics Laboratory, Edwards Air Force Base, California 93523-5000 (1989), available at handle.dtic.mil/100.2/ADA214010. 

cylinder

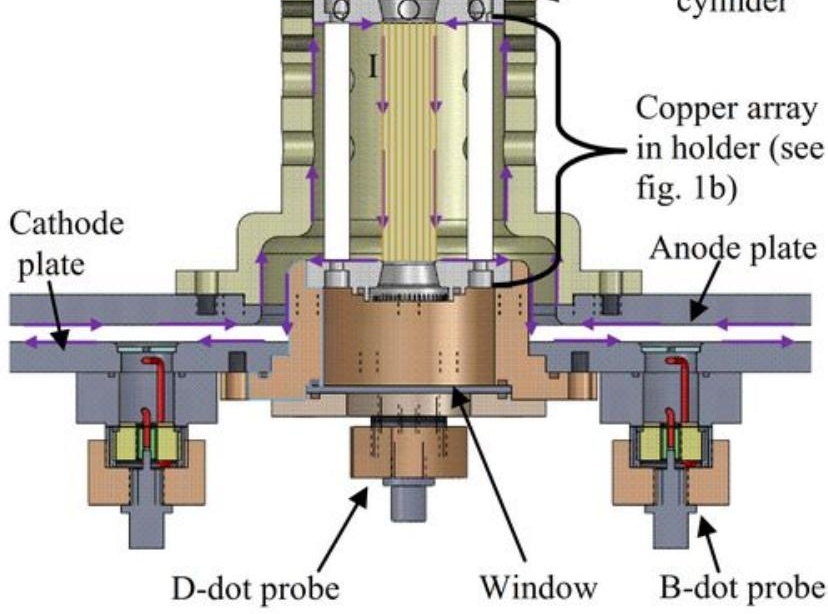

(b)

Notches to position and secure wires

Stainless steel electrode with $10 \mathrm{~mm}$ diameter hole (same at bottom)

$60 \times 130 \mu \mathrm{m}$ copper wires

Delrin spacers 


\section{$532 \mathrm{~nm} 6.5 \mathrm{~W}$ laser, inc}

high speed shutter

I

I $200 \mu \mathrm{m}$

optic $1^{\text {st }}$ stage optical telescope
Beamsplitting and high mag telescopes

I fibre

I

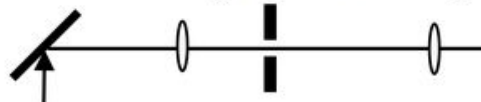

Array in water

1 Collimation optics

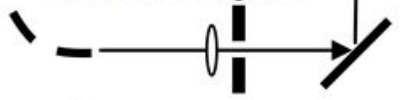

Framing camera
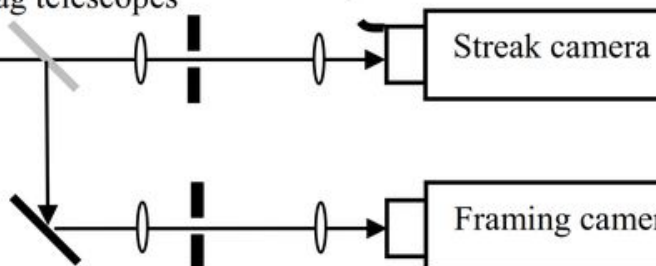

Faming camera
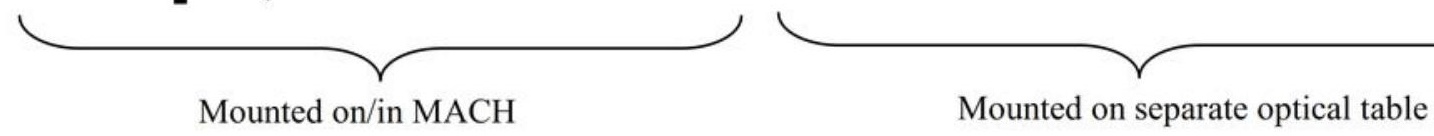


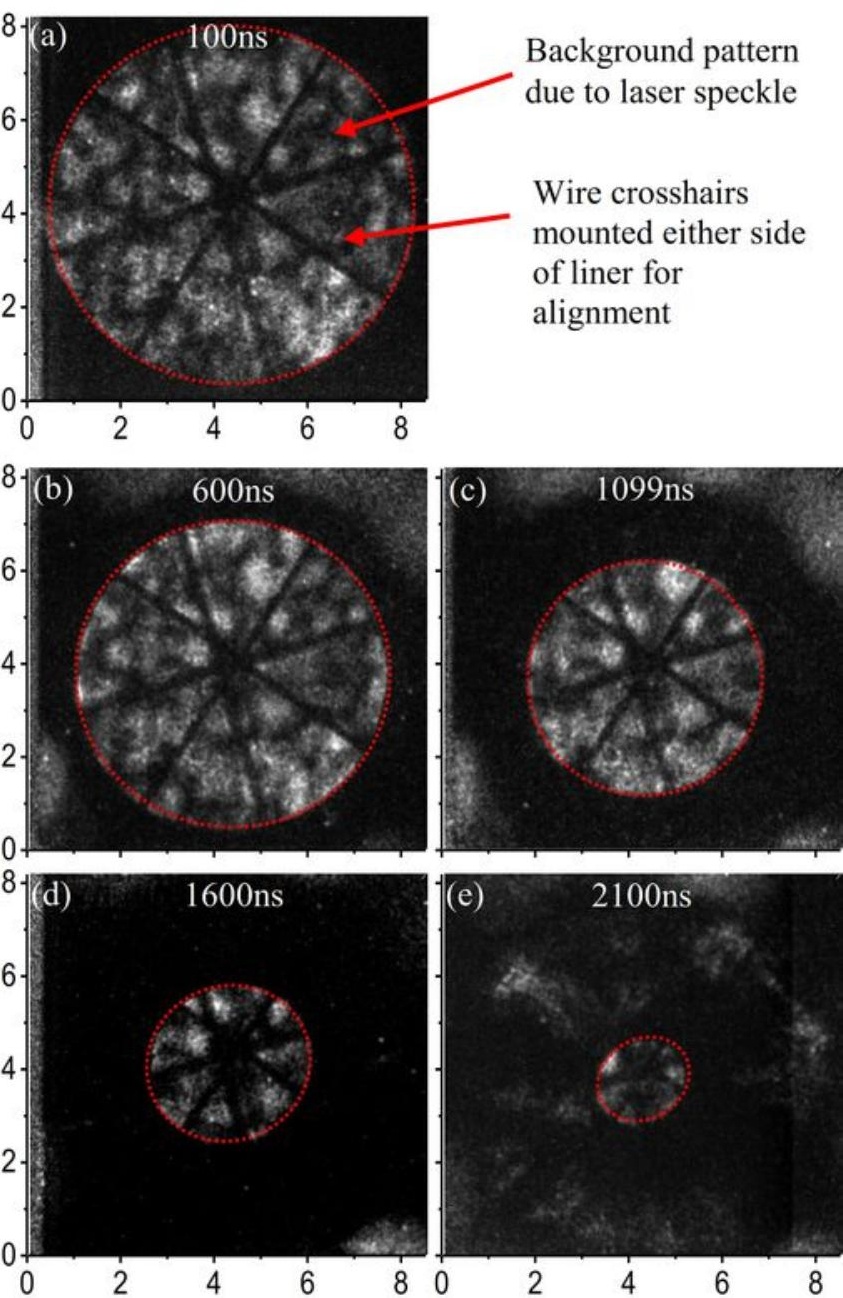




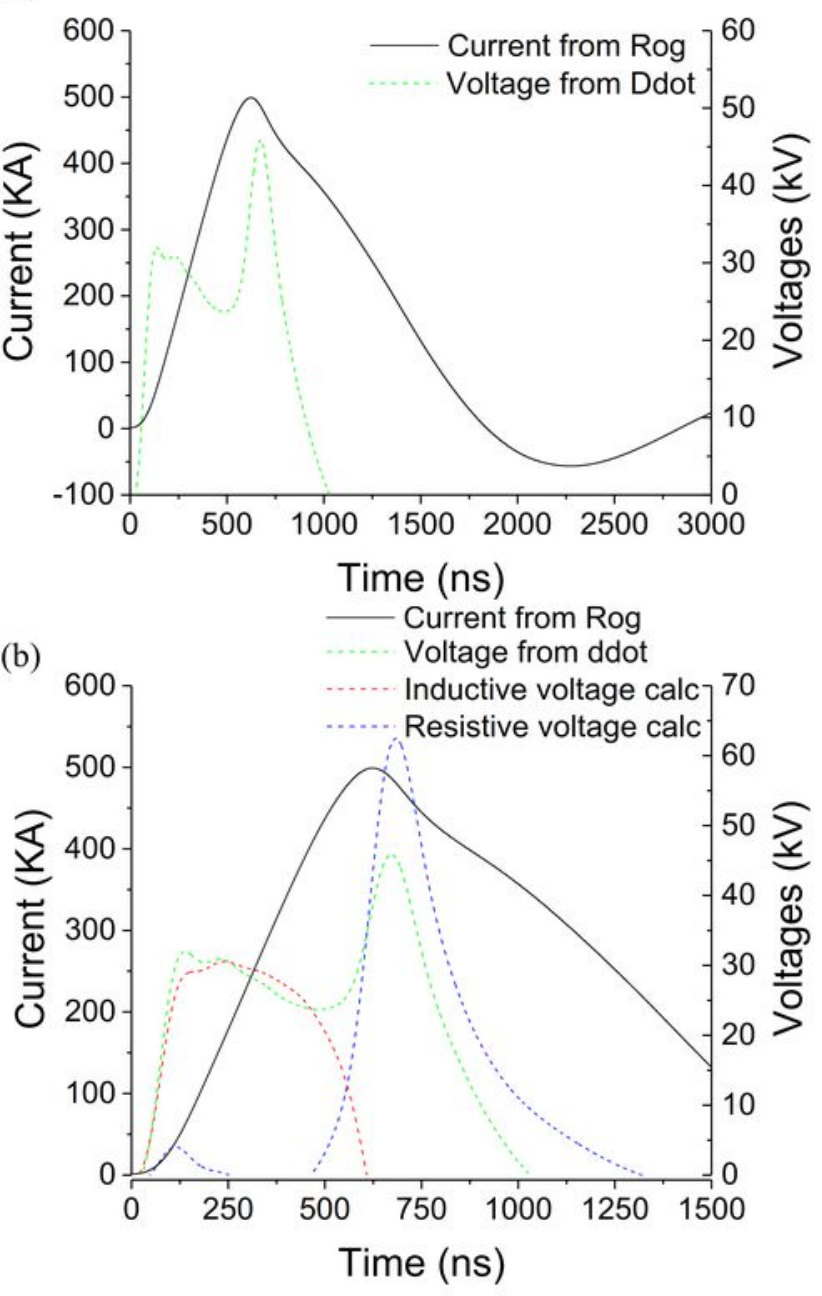

(c)

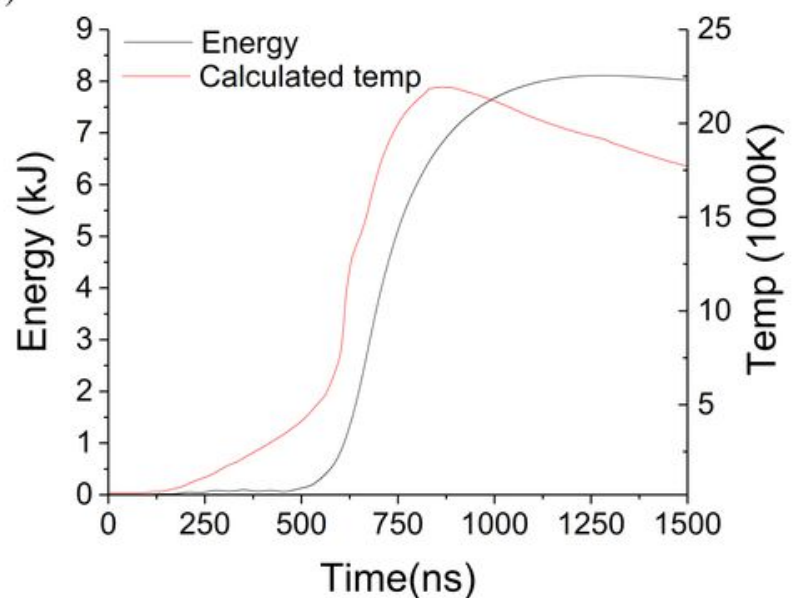




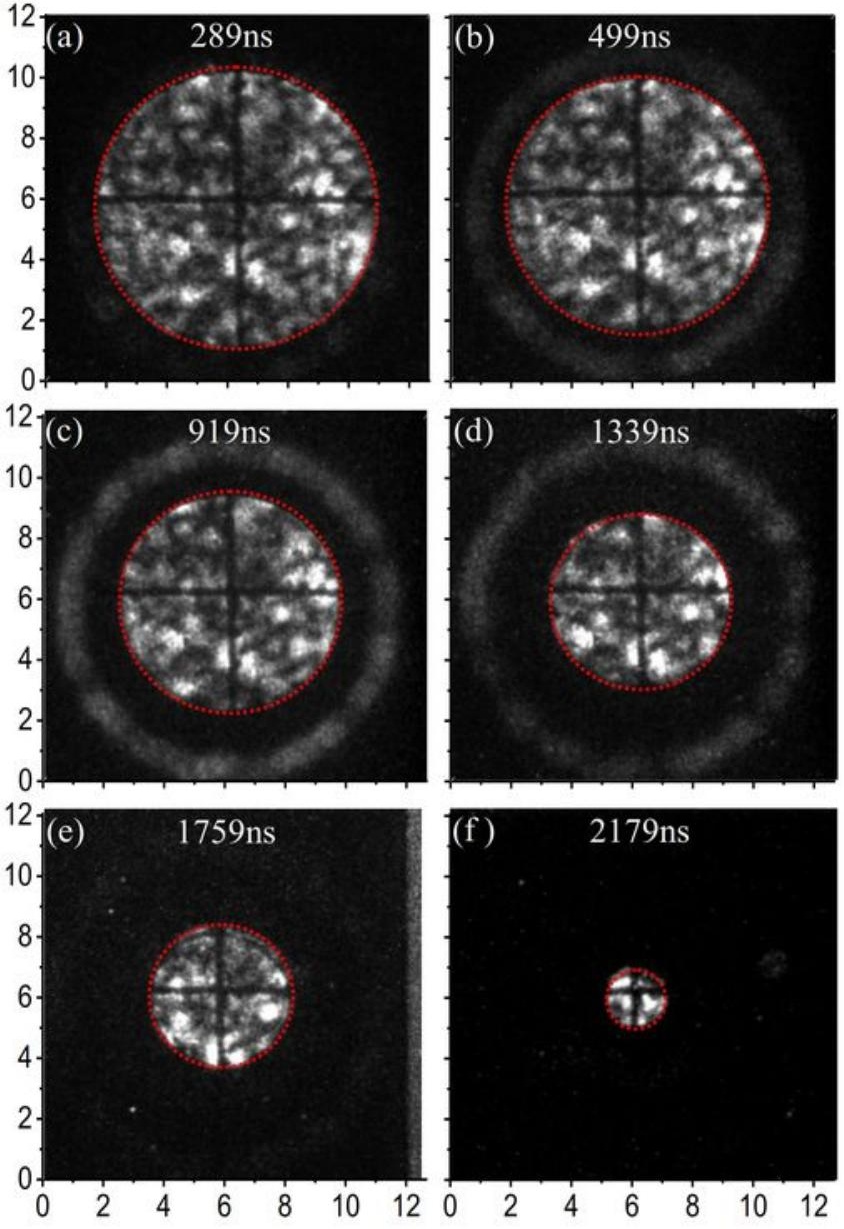

(g)

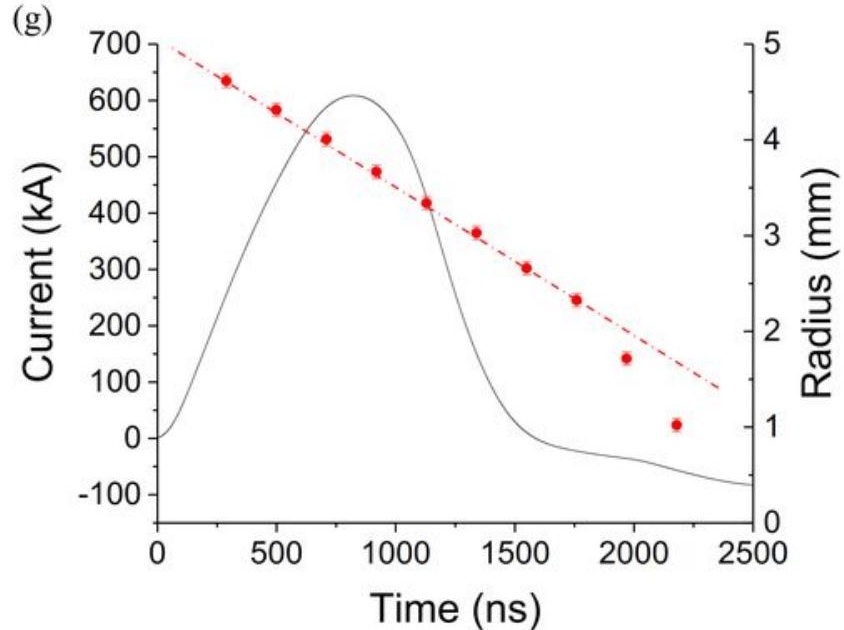






0.6 (e)

0.2

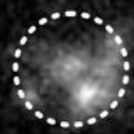

All emission is

0.4

0.0

0.0

$0.2 \quad 0.4$

0.6

from within $200 \mu \mathrm{m}$ diameter circle 


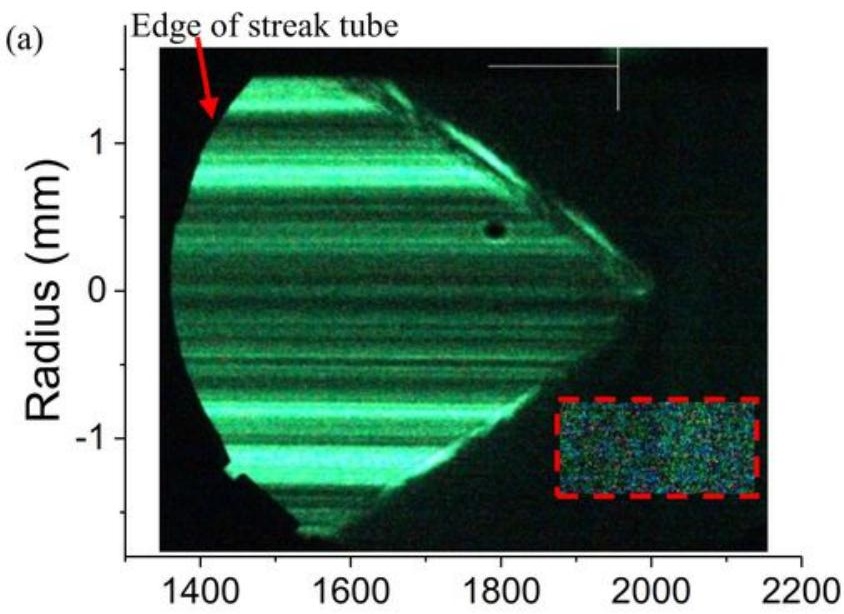

Time (ns)
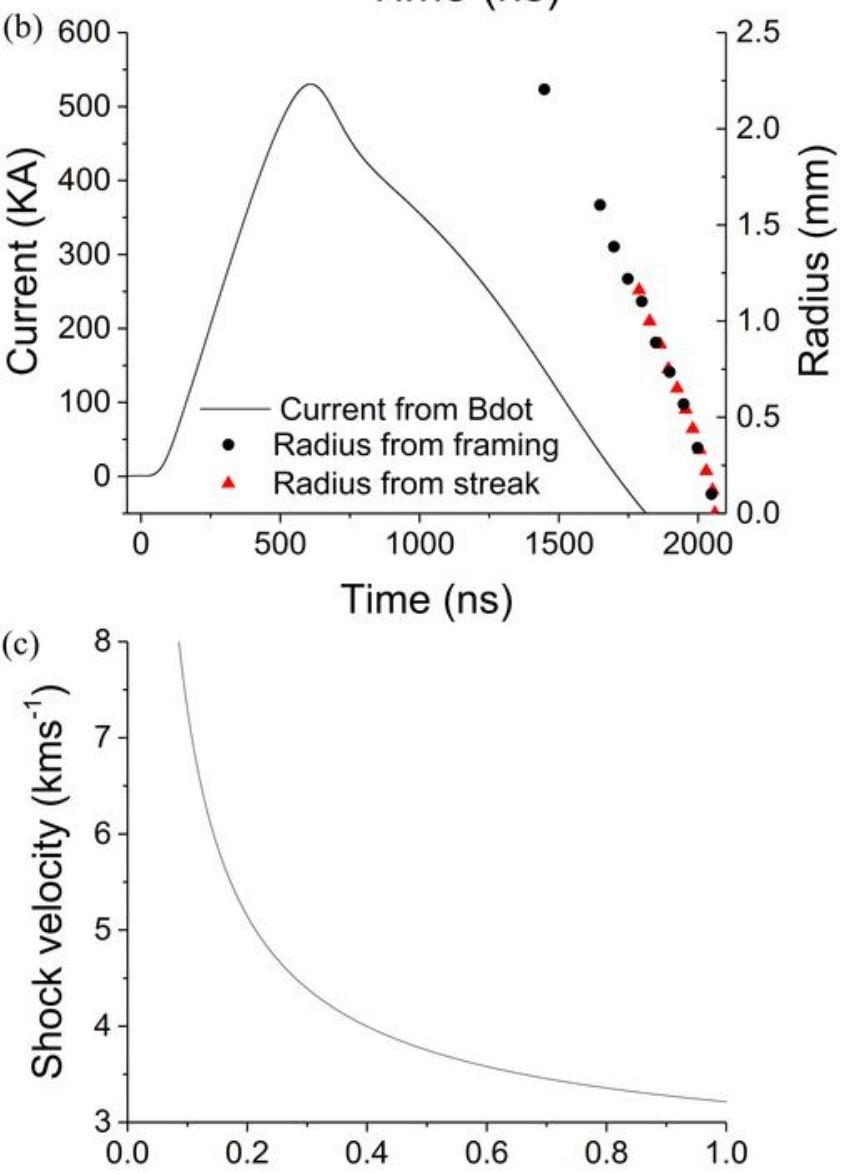

Radius of shock (mm) 
